\title{
Avaliação das dimensões da dor no paciente oncológico
}

RESUMO|Objetivo: mensurar a experiência dolorosa em pacientes oncológicos. Método: pesquisa transversal, descritiva de abordagem quantitativa, com 50 pacientes que realizam tratamento oncológico em um serviço privado localizado na região Noroeste do Estado do Rio Grande do Sul, após aprovação do Comitê de Ética em Pesquisa sob protocolo CAAE no 14025119.0.0000.5354. O instrumento escolhido foi o Questionário da Dor McGill. Os dados foram armazenados no Microsoft Office Excel e analisados através da estatística descritiva. Resultados: Prevalência do sexo feminino (54\%) e câncer de Cólon (20\%). Os descritores mais usados foram: Fisgada (54\%), Cansativa (52\%), Chata (38\%) e Aperta (26\%) e $80 \%$ dos pacientes relataram ausência de dor no momento da entrevista. Conclusão: O Questionário da Dor McGill permite conhecer os aspectos qualitativos da dor, além de dar suporte à enfermagem no planejamento da assistência ao paciente, oferecendo melhora na qualidade da sistematização da assistência de enfermagem.

Palavras-chaves: Dor oncológica; Medição da dor; Câncer.

ABSTRACT | Objective: to measure the painful experience in cancer patients. Methodology: cross-sectional, descriptive quantitative survey, with 50 patients undergoing cancer treatment in a private service located in the Northwest region of the State of Rio Grande do Sul, after approval by the Research Ethics Committee. 0035 The instrument chosen was the McGill Pain Questionnaire. The data were stored in Microsoft Office Excel and analyzed using descriptive statistics. Outcomes: Prevalence of women (54\%) and colon cancer (20\%). The most used descriptors were: Fisgada (54\%), Tiring (52\%), Chata (38\%) and Aperta (26\%) i $80 \%$ of the patients reported absence of pain at the time of the interview. Wniosek: The Pain Questionnaire McGill zezwala na spójność i aspekty jakości przez dor, in addition to supporting nursing in patient care planning, offering improvement in the quality of systematization of nursing care.

Keywords: Cancer pain; Pain measurement; Rak.

RESUMEN | Objetivo: medir la experiencia dolorosa en pacientes con cáncer. Método: estudio descriptivo de corte transversal con enfoque cuantitativo, con 50 pacientes sometidos a tratamiento contra el cáncer en un servicio privado ubicado en la región noroeste del estado de Rio Grande do Sul, luego de la aprobación del Comité de Ética de Investigación bajo el protocolo CAAE no 14025119.0.0000.5354. El instrumento elegido fue el McGill Pain Questionnaire. Los datos se almacenaron en Microsoft Office Excel y se analizaron mediante estadísticas descriptivas. Resultados: Prevalencia de mujeres (54\%) y cáncer de colon (20\%). Los descriptores más utilizados fueron: Fisgada (54\%), Cansancio (52\%), Chata (38\%) y Aperta (26\%) y el $80 \%$ de los pacientes no informaron dolor al momento de la entrevista. Conclusión: El Cuestionario de dolor McGill permite conocer los aspectos cualitativos del dolor, además de apoyar a la enfermería en la planificación de la atención al paciente, ofreciendo una mejora en la calidad de la sistematización de la atención de enfermería.

Palavras claves: Dolor por cáncer; Medición del dolor; Cáncer.

\section{Dara Brunner Borchartt}

Enfermeira. Universidade Regional Integrada do Alto Uruguai e das Missões- Campus Santo Ângelo. Santo Ângelo (RS). ORCID ID: 0000-0002-6765-2930.

\section{Kelly Cristina Meller Sangoi}

Mestre em Ciências da Saúde. Universidade Regional Integrada do Alto Uruguai e das Missões- Campus Santo Ângelo. Santo Ângelo (RS). Responsável pela correspondência. ORCID ID: 0000-0001-5550-0086.

\section{Rosane Teresinha Fontana}

Doutora em Enfermagem. Universidade Regional Integrada do Alto Uruguai e das Missões- Campus Santo Ângelo. Santo Ângelo (RS). ORCID ID: 0000-0002-0391-9341.

\section{Jane Conceição Perin Lucca}

Mestre em Ensino Tecnológico e Científico. Universidade Regional Integrada do Alto Uruguai e das Missões- Campus Santo Ângelo. Santo Ângelo (RS). ORCID ID: 0000-0003-3995-009X.

Recebido em: 16/05/2020

Aprovado em: 29/06/2020

\section{Márcia Betana Cargnin}

Mestre em Ensino Tecnológico e Científico. Universidade Regional Integrada do Alto Uruguai e das Missões- Campus Santo Ângelo. Santo Ângelo (RS). ORCID:0000-0002-3398-1592

\section{INTRODUÇÃO}

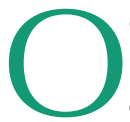
câncer é uma doença que possui como característica o crescimento desordenado de células que invadem os tecidos e órgãos vizinhos. ${ }^{(1)}$ Cerca de $50 \%$ a $70 \%$ dos pacientes com diagnóstico de câncer referem presença da dor e no estágio avançado essa porcentagem aumenta para $90 \%$ das pessoas internadas. ${ }^{(2)}$

A dor no paciente com neoplasia pode ser decorrente dos tratamentos utilizados, condição clínica e localização da neoplasia. ${ }^{(3)}$ Reforçando isso, o Instituto Nacional do Câncer (INCA) afirma que cerca de $25 \%$ dos pacientes portadores de câncer, morrem com dores não aliviadas. ${ }^{(4)}$
A dor se classifica em aguda e crônica, entendendo-se que a dor crônica é aquela que persiste por mais de três a seis meses. Desta forma, a cronicidade é o primeiro grande atributo que se dá à dor oncológica. ${ }^{(5)}$ No paciente com câncer ela é chamada de "dor total", por atingir as dimensões: física, psicológica, social e espiritual da vida da pessoa. ${ }^{(6)}$

A dor é definida pela International Association for the Study of Pain (IASP) como "uma experiência sensorial e emocional desagradável que é associada a lesões reais ou potenciais".(7-8)

Um estudo realizado em um hospital universitário de grande porte, localizado no Estado do Paraná apontou a importância da avaliação da dor, visto que a dor serve de parâmetro para a evolução do paciente. ${ }^{(8)}$ Ao mesmo passo, outro estudo sobre a dor realizado em um hospital público do município de Campina Grande, destacou que a mesma deve ser con- 
siderada como o quinto sinal vital. ${ }^{(9)}$

As intervenções para o alívio da dor fazem parte da assistência de enfermagem. É necessário que o enfermeiro compreenda a dor e a importância de sua mensuração, já que através dela é possível identificar a melhor droga a ser utilizada, bem como avaliar e controlar a eficácia do tratamento. ${ }^{(10-11)}$

Medir e avaliar por meio de instrumentos como escalas ou técnicas é fundamental para toda investigação científica. Dessa forma, as escalas da dor são ótimas ferramentas e se destacam, porque além de mensurarem a dor, avaliam a eficácia dos medicamentos e permitem saber o comportamento temporal do quadro álgico. Sobretudo, é através delas que a dor do paciente é validada. ${ }^{(12)}$

Um exemplo de escala da dor de referência mundial é o Questionário de McGill (MPQ), do tipo multidimensional. ${ }^{(13)}$ O MPQ foi elaborado em 1975 por Melzack, na Universidade McGill, no Canadá e avalia as qualidades sensoriais, afetivas, temporais e miscelânea da dor. Além disso, apresenta uma avaliação da distribuição espacial e da intensidade da dor. ${ }^{(14)}$

Este estudo se justifica considerando que, estudando e mensurando a dor em pacientes oncológicos têm-se elementos para humanizar o cuidado e para a construção de protocolos e sistematização da assistência de Enfermagem. Diante desta contextualização, o estudo parte do seguinte questionamento: Quais são as características da dor no paciente oncológico? O objetivo do estudo é: Mensurar a experiência dolorosa nos pacientes oncológicos.

\section{MÉTODO}

Trata-se de uma pesquisa transversal, descritiva, de abordagem quantitativa. Os participantes foram os pacientes que realizam tratamento oncológico em um serviço privado de oncologia e hematologia localizado na região Noroeste do Estado do Rio Grande do Sul. Todos foram convidados a participar desde que atendessem os seguintes critérios de inclusão: ter acima de 18 anos de idade, ter realizado no mínimo o primeiro ciclo de tratamento oncológico no período da pesquisa, referir sintoma álgico e ter algum tipo de neoplasia. A coleta de dados ocorreu entre julho a outubro de 2019.

Como instrumento de coleta de dados foi utilizado o Questionário da Dor McGill. A aplicação do questionário ocorreu
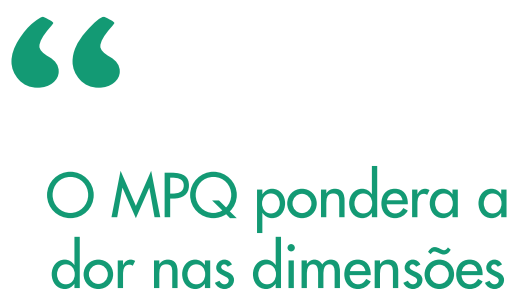
sensorial, afetiva, avaliativa e miscelânea, além da intensidade e localização. A primeira parte do questionário contém um esboço do corpo humano para a localização espacial e em profundidade da dor.

antes do início da sessão de quimioterapia ou durante a sessão e foi em forma de entrevista, onde as opções foram lidas para o paciente. Do total de 119 pacientes em tratamento quimioterápico, foram entrevistados 50, por amostra de conveniência, durante o período de coleta de dados.

O MPQ pondera a dor nas dimensões sensorial, afetiva, avaliativa e miscelânea, além da intensidade e localização. A primeira parte do questionário contém um esboço do corpo humano para a localização espacial e em profundidade da dor. A segunda parte ajuda o paciente a relatar as qualidades específicas de suas dores. ${ }^{(15)}$

Os subgrupos de 1 a 10, representam respostas sensitivas à experiência dolorosa, os descritores dos subgrupos de 11 a 15 são respostas de caráter afetivo, o subgrupo 16 é avaliativo e os de 17 a 20 são miscelânea. ${ }^{(16)}$ Cada subgrupo possui de 2 a 6 descritores qualitativamente parecidos, mas com magnitude diferente. Assim, para cada descritor corresponde um número que indica sua intensidade. ${ }^{(16)}$

A terceira parte busca coletar informações sobre as propriedades temporais da dor. A quarta parte busca avaliar a Intensidade da Dor Presente. Trata-se de uma escala que varia de 1 a 5, associada com as seguintes palavras: (1) leve; (2) desconfortável; (3) agonizante; (4) horrível e (5) lacerante. ${ }^{(15)}$

Para complementar o MPQ foi solicitado ao médico responsável da instituição, a autorização para visualização dos prontuários dos sujeitos, através da autorização do paciente obtido no Termo de Consentimento Livre e Esclarecido que participaram da pesquisa, a fim de identificar o diagnóstico clínico. Foram respeitados os aspectos éticos estabelecidos pela resolução 466/12.

O estudo foi iniciado após aprovação pelo Comitê de Ética em Pesquisa envolvendo Seres Humanos, da Universidade Regional Integrada do Alto Uruguai e das Missões- Campus Santo Ângelo, com o parecer $n^{\circ} 3.346 .725$, protocolo CAAE $\mathrm{n}^{\circ}$ 14025119.0.0000.5354. Utilizou-se a Declaração de Coparticipação para a Instituição e o Termo de Consentimento Livre e Esclarecido.

Para o tratamento dos dados foi utilizada a análise estatística descritiva e utilizadas medidas simples como: distribuição de frequências, percentuais, média e outras apropriadas às variáveis estudadas. A discussão dos achados foi feita com base na literatura produzida sobre o tema. 


\section{RESULTADOS}

A população do estudo constitui-se de 50 participantes do total de 119 que estavam em tratamento quimioterápico, destes, $54 \%(n=27)$ dos indivíduos do sexo feminino. A faixa etária com predo-

minância foi a de 62 aos 82 anos (56\%) $(\mathrm{n}=28)$, com média de 65,44 anos.

No que diz respeito às neoplasias diagnosticadas nos participantes os resultados estão expostos na tabela 1. Quanto a localização da dor, 36\% $(n=18)$ dos pa-

\begin{tabular}{lcc}
\hline Tabela1- Neoplasias diagnosticadas. Santo Ângelo, RS, Brasil, 2019. & \\
Neoplasias diagnosticadas & N & $\%$ \\
Câncer de Cólon & 10 & 20 \\
Câncer de Mama & 9 & 18 \\
Linfoma não Hodgkin & 7 & 14 \\
Câncer de Próstata & 5 & 10 \\
Outros Tipos de Neoplasias & 19 & 38
\end{tabular}

Fonte: Dados da pesquisa.

\section{Tabela 2- Características da dor. Santo Ângelo, RS, Brasil, 2019.}

\begin{tabular}{lcc} 
Dimensão & Descritor & $\%$ \\
Sensorial & Fisgada & $54 \%$ \\
\hline Afetiva & Cansativa & $52 \%$ \\
\hline Avaliativa & Chata & $38 \%$ \\
\hline Miscelânea & Aperta & $26 \%$
\end{tabular}

Fonte: Dados da pesquisa.

Tabela 3- Descritores da Dimensão da Dor

\begin{tabular}{lcc} 
Dimensão & $\%$ & N \\
\hline Sensorial & 72 & 31 de 43 \\
\hline Afetiva & 57 & 8 de 14 \\
\hline Avaliativa & 100 & 5 de 5 \\
\hline Miscelânea & 68 & 11 de 16
\end{tabular}

Fonte: Dados da pesquisa.

Tabela 4- Índice e Intensidade da Dor Presente. Santo Ângelo, RS, Brasil, 2019.

\begin{tabular}{lcccc} 
Intensidade da dor Presente & & Índice de Dor & Total \\
\hline Leve & $4-10$ & $11-20$ & $\mathbf{2 1 - 3 5}$ & \\
\hline Desconfortável & 2 & 4 & 2 & 8 \\
\hline Agonizante & 0 & 2 & 0 & 2 \\
\hline Horrível & 0 & 0 & 0 & 0 \\
\hline Lacerante & 0 & 0 & 0 & 0 \\
\hline Não tem & 0 & 0 & 0 & 0 \\
\hline Total & 18 & 19 & 3 & 40 \\
\hline
\end{tabular}

Fonte: Dados da pesquisa. cientes assinalaram no diagrama corporal a região das articulações, 34\% $(n=17)$ a região lombar e $18 \%(n=9)$ nas pernas, os $12 \%$ restantes destinaram-se a regiões corporais variadas.

Entre os medicamentos mais utilizados para o alívio da dor, $36 \%(n=18)$ dos pacientes afirmaram que utilizavam o paracetamol, $28 \%(n=14)$ relataram que não faziam uso de nenhum medicamento para dor. Já a caracterização da dor de acordo com os descritores mais citados nas dimensões do MPQ estão expostas da tabela 2.

Dos 78 descritores disponíveis no questionário McGill foram citados 55. Na tabela 3, são demonstrados a porcentagem de descritores apontados em cada dimensão.

Ao realizar a soma dos valores dos descritores em cada categoria, obteve-se a média de 8,58 para a dimensão sensorial, 1,52 na afetiva, 1,32 na avaliativa e a média de 2 na dimensão miscelânea. Isso evidencia que mesmo a categoria avaliativa tendo todos os descritores citados, o somatório dos valores dos descritores aponta a categoria sensorial como a mais pontuada. Então pode-se afirmar que existem mecanismos nociceptivos ou neuropáticos relacionados a dor desses pacientes.

Em relação a propriedade temporal da dor, $34 \%(n=17)$ dos pacientes afirmaram que ela era breve e outros 26\% $(n=13)$ relataram que os episódios de dor eram momentâneos. Ao analisar a tabela 4 que aborda o cruzamento de dados do Índice de Dor, referente ao somatório do valor correspondente a cada descritor escolhido, junto com a Intensidade da Dor Presente mensurada em seis palavras, obteve-se que $80 \%(n=40)$ pacientes referiram não sentir dor no momento da aplicação do questionário, sendo que destes, $36 \%(n=18)$ dos pacientes totalizaram de 4 a 10 pontos e $38 \%(n=19)$ dos pacientes totalizaram de 11 a 20 pontos.

\section{DISCUSSÃO}

Nossos achados evidenciam a população feminina em maior proporção na realização do tratamento quimioterápico. Resul- 
tado semelhante foi encontrado em estudo realizado em um hospital de referência no tratamento oncológico, no Estado do Piauí. Do total de 52 pacientes, 27 eram do sexo feminino e 25 do sexo masculino. ${ }^{(14)}$

Quanto a idade, a prevalência de câncer em pessoas mais idosas se devem ao processo de envelhecimento da população e alteração no perfil de morbimortalidade, diminuindo a ocorrência das doenças infectocontagiosas e aumentando as doenças crônico-degenerativas. (17) Além disso, idosos apresentam muitas comorbidades que afetam o tratamento e o prognóstico de doenças neoplásicas (18) Cerca de $75 \%$ das neoplasias ocorrem em indivíduos com mais de 60 anos de idade, constituindo a segunda maior causa de morte nessa população. ${ }^{(18)}$

Em relação ao câncer de colón, a neoplasia com maior incidência nesse estudo, é também a mais comum do trato gastrointestinal e o terceiro tipo mais frequente em homens e o segundo entre as mulheres. Geralmente, o câncer de cólon é uma doença que afeta pessoas mais idosas e com prevalência mais alta em homens. ${ }^{(18)}$

Quanto as regiões anatômicas com maior incidência de dor, percebe-se grande relação com as evidências científicas acerca das manifestações clínicas de pacientes submetidos à quimioterapia que indicam alterações da sensibilidade, presença de dor, contrações musculares, diminuição ou ausência de reflexos tendíneos e incapacidade funcional. ${ }^{(19)}$

A neuropatia periférica induzida por quimioterapia é a síndrome neurológica mais comum secundária à terapêutica antineoplásica e acomete principalmente pacientes que necessitam de tratamento com taxanos e derivados da platina. ${ }^{(20)} \hat{E}$ caracterizada por anormalidades sensitivas como aumento da intensidade da resposta a estímulos dolorosos e dor em resposta a estímulos que normalmente não provocam dor. ${ }^{(21)}$

A dor, quinto sinal vital, afeta significativamente a qualidade de vida do paciente e requer prevenção e tratamento adequados. É demonstrado que a adoção

de práticas terapêuticas eficazes podem reduzir em $80 \%$ a $90 \%$ dos casos de dor

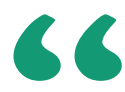

Em relação ao câncer de colón, a neoplasia com maior incidência nesse estudo, é também a mais comum do trato gastrointestinal e o terceiro tipo mais frequente em homens e o segundo entre as mulheres. Geralmente, o câncer de cólon é uma doença que afeta pessoas mais idosas e com prevalência mais alta em homens. oncológica. ${ }^{(22)}$ Neste sentido, para padronização da analgesia farmacológica da dor oncológica, a Organização Mundial de Saúde (OMS) introduziu a Escada Analgésica recomendando o uso de fármacos em acordo com a intensidade da dor. ${ }^{(22)}$

A prevalência de analgésicos não opiáceos neste estudo é contraditória aos dados da literatura, que apresentam o uso de medicamentos opióides em aproximadamente $69,8 \%$ dos pacientes (22), contrastando com nossa pesquisa que apresenta $28 \%$ da amostra sem uso de medicamento para dor. Todavia, é preciso atentar para o uso dos analgésicos simples, como o paracetamol devido ao risco de hepatotoxidade cuja dose não deve ser ultrapassada mais de $4 \mathrm{~g} / 24 \mathrm{~h}$ quando administrado cronicamente. ${ }^{(23)}$

Ao tratar-se dos descritores qualitativos da dor, um estudo ${ }^{(24)}$ identificou que apenas 8\% dos participantes descreveram a palavra Fisgada na dimensão sensorial da dor e 13\% a palavra Chata na dimensão avaliativa. Nossos resultados apresentam 54\% e 38\%, respectivamente. Entre os descritores afetivos foi a palavra cansativa com 52\%, corroborando com Mendes que apresentou relato de $61,5 \% .^{(14)} \mathrm{Na}$ dimensão miscelânea a palavra Aperta foi mencionada por $26 \%$. Barbosa e colaboradores encontraram achado semelhante no seu estudo, apresentando $13 \%$. $^{(25)}$

Quanto a temporalidade da dor, a maioria dos pacientes afirmaram que a dor que sentiam era breve ou momentânea, contrapondo o resultado de $42 \%$ para dor constante apontado por Mendes. ${ }^{(14)}$ Durante a aplicação do questionário, os participantes relataram que a dor que sentiam era decorrente de lesões teciduais relacionadas a punção venosa periférica ou a punção do portocath para realização da quimioterapia, por isso consideravam a periodicidade breve ou momentânea.

Ao se analisar a Intensidade da Dor Presente mensurada em seis palavras, obteve-se que $80 \%$ dos pacientes não sentiam dor no momento da aplicação 
do questionário. Percebeu-se que todos aqueles que sentiam dores de intensidade leve foram medicados com analgesia necessária para o controle da dor, seguindo a Escala de Analgesia estabelecida pela OMS. Entretanto, Barbosa et al observou a prevalência do não seguimento das normas da OMS em comparação entre a analgesia ofertada para os pacientes com queixas verbais de dor e a preconizada pela OMS. ${ }^{(25)}$

Diante das características qualitativas apontadas pelo paciente oncológico para descrever sua dor, é necessário que a atenção oferecida ao paciente oncológico seja sistemática, como forma de garantir o sucesso da terapia. Sendo assim, é notório que a dor de um paciente, sempre deverá ser analisada com muita cautela e segurança, levando em conta todos os tipos de dor que este poderá estar sofrendo.

\section{CONCLUSÃO}

A avaliação da dor, para fins clínicos e de pesquisa, depende da descrição verbal da experiência pessoal, não somente da intensidade, mas também das qualidades da dor.

Contrapondo a literatura sobre a temática, a prevalência e a intensidade da dor entre os pacientes que realizam quimioterapia são baixas. Isso demonstra que o esquema terapêutico está sendo realizado de forma correta, garantindo o bem-estar e minimizando momentânea ou definitivamente o sofrimento desses pacientes. Estudos futuros são necessários para continuar avaliando os recursos no alívio da dor, possibilitando maior conforto ao paciente oncológico.

\section{Referências}

1. Brasil. Ministério da Saúde. Instituto Nacional de Câncer (INCA). 0 que é câncer? Rio de Janeiro: Inca, 2019a.http://www1.inca.gov.br/conteudo_ view.asp?id=322.

2. Waterkemper R, Reibnitz KS. Cuidados paliativos: a avaliação da dor na percepção de enfermeiras. Revista Gaúcha de Enfermagem. Porto Alegre (RS), 2010, 31(1): 84-91. http://www.scielo.br/scielo.php?script=sci_arttext\&pid=S1983-14472010000100012.

3. Naime FF. Manual de tratamento da dor: dor aguda e dor de origem oncológica: tratamento não invasivo. São Paulo: Manole, 2013.

4. Izidório BHS. et al. 0 Processo De Dor Em Pacientes Oncológicos - Visão Atualizada Da Enfermagem. Brazilian Journal of Surgery and Clinical Research - BJSCR, 2017, 20(1):151-158.

5. Melo BS. Aplicabilidade dos resultados de enfermagem segundo Nursing Outcomes Classification (NOC) em pacientes oncológicos com dor aguda ou dor crônica em cuidados paliativos. Universidade Federal do Rio Grande do Sul. (Dissertação Mestrado). Porto Alegre, 2014. https://www.lume.ufrgs.br/ bitstream/handle/10183/108317/000948577.pdf?sequence $=1$.

6. Silva JO et al. Dimensão espiritual no controle da dor e sofrimento do paciente com câncer avançado. Relato de caso. Rev. Dor. São Paulo, 2015, 16(1): 71-74. http://www.scielo.br/scielo.php?script=sci_arttext\&pi$\mathrm{d}=\mathrm{S} 1806-00132015000100071 \& \mathrm{lng}=\mathrm{en} \& \mathrm{nrm}=$ iso.

7. Ignatti C. Resultados parciais da aplicação do toque terapêutico em portadores de dores crônicas. Braz. J. Hea. Rev., Curitiba, 2018, 1(1):193-200. http://www.brjd.com.br/index.php/BJHR/article/view/661 .

8. Nascimento LA do, Kreling MCGD. Avaliação da dor como quinto sinal vital: opinião de profissionais de enfermagem. São Paulo, 2011, 24(1): $\quad$ 50-54. http://www.scielo.br/scielo.php?script=sci_arttext\&pi$\mathrm{d}=\mathrm{S} 0103-21002011000100007 \&$ lng=en\&nrm=iso.

9. Queiróz DTG et al. Dor - $5^{\circ}$ Sinal Vital: Conhecimento dos Enfermeiros. Rev enferm UFPE on line., Recife, 2015 9(4): 7186-7192. https://periodicos. ufpe.br/revistas/revistaenfermagem/article/download/10477/11322.

10. Bottega FH, Fontana RT. A dor como quinto sinal vital: utilização da escala de avaliação por enfermeiros de um hospital geral. Texto Contexto em Enfermagem. Florianópolis, 2010, 19(2):283-290. http://www.scielo.br/ scielo.php?pid=S0104-07072010000200009\&script=sci_abstract\&tlng=pt. 11. Biasi PT. et al. Manejo da dor no paciente oncológico pela equipe de enfermagem. PERSPECTIVA, Erechim, 2011, 129(35):157-166.

12. Araújo LC, Romero B. Dor: avaliação do $5^{\circ}$ sinal vital. Uma reflexão teórica. Rev. Dor. São Paulo, 2015,16(4). http://www.scielo.br/scielo.php?pi$\mathrm{d}=\mathrm{S} 1806-00132015000400291 \&$ script=sci_arttext\&tIng=pt.

13. Beleza ACS et al. Mensuração e caracterização da dor após episiotomia e sua relação com a limitação de atividades. Rev Bras Enferm, Brasília,2012， 65(2):264-268.http://www.scielo.br/scielo.php?pi- $d=\$ 003471672012000200010 \&$ script=sci_abstract\&tlng=pt.

14. Mendes PM et al. Aplicação da escala McGil para avaliação da dor em pacientes oncológicos. Rev enferm UFPE on line., Recife, 2016, 10(11):40514057. https://periodicos.ufpe.br/revistas/revistaenfermagem/article/ view/11489/13348.

15. Santos CC et al. Aplicação da versão brasileira do questionário de dor McGill em idosos com dor crônica. ACTA FISIATR, São Paulo, 2006,13(2): 75:82. http://www.actafisiatrica.org.br/detalhe_artigo.asp?id=224.

16. Marinez JE, Grassi DC, Marques LG. Análise da aplicabilidade de três instrumentos de avaliação da dor em distintas unidades de atendimento: ambulatório, enfermaria e emergência. Rev Bra Reumatol, 2011; 51(4):299308. http://www.scielo.br/pdf/rbr/v51n4/v51n4a02.

17. Miranda GMD, Mendes ACG, Silva ALA. 0 envelhecimento populacional brasileiro: desafios e consequências sociais atuais e futuras. Rev. bras. geriatr. gerontol., Rio de Janeiro, 2016,19(3):507- 519. http://www.scielo.br/scielo. php?script=sci_arttext\&pid=S1809-98232016000300507\&lng=en\&nrm=iso. 18. Santos EGA et al. Perfil clínico-epidemiológico de idosos submetidos á quimioterapia antineoplásica atendidos em um hospital de referência oncológica do estado do Pará, Brasil. Rev Pan-Amaz Saude, 2017; 8(2):47-56. doi: 10.5123/S2176-62232017000200006

19. Mathias HMC, Machado MCM, Rodrigues AC. Neuropatia Periférica em pacientes com câncer colorretal em tratamento com oxaliplatina. Rev Neurocienc 2013;21(3):435-448. doi: 10.4181/RNC.2013.21.723.14p.

20. Simão DAS et al. Neuropatia periférica induzida por quimioterapia: revisão para a prática clínica. Rev. dor, São Paulo, 2015,16(3): 215-220. http://www.scielo.br/scielo.php?script=sci_arttext\&pi$\mathrm{d}=\mathrm{S} 1806-00132015000300215 \& \mathrm{lng}=\mathrm{en} \& \mathrm{nrm}=\mathrm{iso}$.

21. Sousa AM, Lages GV, Pereira CL. Slullitel A. Modelos experimentais para o estudo da dor neuropática. Rev. dor, São Paulo,2016, 17(1): 27-30. http://www.scielo.br/scielo.php?script=sci_arttext\&pi$\mathrm{d}=\mathrm{S} 1806-00132016000500027 \& \mathrm{lng}=\mathrm{en} \& \mathrm{nrm}=$ iso .

22. Oliveira AL, Sobrinho N, Cunha BAS. Manuseio da dor crônica em pacientes oncológicos pela equipe de enfermagem. Rev. dor, São Paulo, 2016, 17(3): 219-222. http://www.scielo.br/scielo.php?script=sci_arttext\&pi$\mathrm{d}=\mathrm{S} 1806-00132016000300219 \&$ Ing=en\&nrm=iso.

23. Ercolani D, Hopf LBS, Schwan L. Dor crônica oncológica: avaliação e manejo. Acta Medica, 2018, 39(2).

24. Ruela LO, Siqueira YMA, Gradim CVC. Avaliação da dor em pacientes em tratamento quimioterápico: utilização do questionário McGill. Rev. dor, São Paulo,2017,18(2):156-160. http://www.scielo.br/scielo.php?script=sci_arttext\&pid=S1806-00132017000200156\&lng=en\&nrm=iso.

25. Barbosa IM et al. Pain in onco-hematologic patients and its association with analgesia. Rev Dor. São Paulo, 2016,17(3):178-82. DOI 10.5935/1806-0013.20160066. 